in vivo 35 : $1041-1049$ (2021)

doi:10.21873/invivo.12348

\title{
Neo-Bioscore in Guiding Post-surgical Therapy in Patients With Triple-negative Breast Cancer Who Received Neoadjuvant Chemotherapy
}

\author{
YORIKO HASEGAWA ${ }^{1}$, NOBUAKI MATSUBARA ${ }^{1}$, TAKAHIRO KOGAWA ${ }^{1}$, YOICHI NAITO ${ }^{1}$, \\ KENICHI HARANO ${ }^{1}$, AKO HOSONO ${ }^{1}$, TATSUYA ONISHI ${ }^{2}$, TAKASHI HOJO ${ }^{2}$, \\ MOTOTSUGU SHIMOKAWA ${ }^{3}$ and TORU MUKOHARA ${ }^{1}$ \\ ${ }^{1}$ Department of Breast and Medical Oncology, National Cancer Center Hospital East, Kashiwa, Japan; \\ ${ }^{2}$ Department of Breast Surgery, National Cancer Center Hospital East, Kashiwa, Japan; \\ ${ }^{3}$ Department of Biostatistics, Yamaguchi University Graduate School of Medicine, Ube, Japan
}

\begin{abstract}
Aim: Patients with triple-negative breast cancer (TNBC) who have not achieved pathological complete response ( $p C R$ ) after neoadjuvant chemotherapy (NAC) were considered for adjuvant capecitabine. This study was to explore the utility of the Neo-Bioscore in guiding post-surgical therapy in TNBC. Patients and Methods: The Neo-Bioscore was calculated for patients with non-metastatic primary breast cancer who received NAC at National Cancer Center Hospital East, Japan. Results: A total of 329 patients were evaluated. The Neo-Bioscore stratified prognosis after NAC better than clinical or pathological stage. The Neo-Bioscore performed well in the selection of patients with TNBC with excellent prognoses despite non-pCR; no death was observed in patients who had a Neo-Bioscore of 2, the lowest score in those with TNBC. Conclusion: The Neo-Bioscore can improve the prognostic stratification of patients after NAC for breast cancer over clinical and pathological staging and may enable the identification of patients with non-pCR TNBC who can avoid additional adjuvant chemotherapy.
\end{abstract}

Neoadjuvant chemotherapy (NAC) has been adopted as the standard of care for early and locally advanced breast cancer for the past two decades. While NAC does not improve disease-free survival (DFS) or overall survival (OS) compared

This article is freely accessible online.

Correspondence to: Toru Mukohara, MD, D Med Sci, Department of Breast and Medical Oncology, 6-5-1 Kahiwanoha, Kashiwa 2778577, Japan. Tel: +81 471331111, Fax: +81 471346922, e-mail: tmukohar@east.ncc.go.jp

Key Words: Neo-Bioscore, neoadjuvant chemotherapy, triplenegative breast cancer, prognosis. with adjuvant chemotherapy composed of the same treatment regimen (1-3), NAC has several advantages over adjuvant chemotherapy. Firstly, it increases the chance for breastconserving surgery even when mastectomy is the only option at diagnosis. Secondly, clinical response can be monitored by measuring tumor size during NAC, which prevents giving ineffective chemotherapy to the patient. Thirdly, the effectiveness of the particular chemotherapy can be evaluated pathologically. Pathological complete response (pCR) is generally associated with a good prognosis (4). Until recently, however, pathological response was used only as a prognostic marker and not as a determinant of treatment after surgery.

Two randomized phase III trials provided a paradigm shift showing that the prognosis of patients who have residual disease (non-pCR) can be improved by giving additional treatment over standard therapy $(5,6)$. The first trial was CREATE $X$, in which patients with human epidermal growth factor receptor 2 (HER2)-negative breast cancer who had residual disease after standard NAC were randomized to standard therapy as the control arm or standard therapy plus capecitabine as the experimental arm (5). Capecitabine treatment was found to significantly improve DFS and OS [hazard ratio $(\mathrm{HR})=0.70,95 \%$ confidence interval $(\mathrm{CI})=0.53$ 0.92 ; and $0.59,95 \% \mathrm{CI}=0.39-0.90$, respectively], along with increased incidence of diarrhea, mucositis, and hand-foot syndrome. The benefit of adding capecitabine was particularly prominent in patients with triple-negative breast cancer (TNBC) (DFS: $69.8 \%$ in the capecitabine group vs. $59.1 \%$ in the control group; $\mathrm{HR}=0.58,95 \% \mathrm{CI}=0.39-0.87)$. Based on these results, the National Comprehensive Cancer Network guideline recommends adjuvant capecitabine only in patients with TNBC who have residual disease after NAC (7). The second trial was KATHERINE, in which patients with HER2-positive breast cancer who had residual disease after standard NAC containing trastuzumab were randomized to continuing trastuzumab as the 
Table I. Point assignments for Neo-Bioscore staging systems.

\begin{tabular}{lcc}
\hline & Subgroup & Neo-Bioscore points \\
\hline Clinical stage & I & 0 \\
& IIA & 0 \\
IIB & 1 \\
IIIA & 1 \\
Pathological stage & IIIB & 2 \\
& IIIC & 2 \\
& 0 & 0 \\
I & 0 \\
IIA & 1 \\
IIB & 1 \\
Tumor marker & IIIA & 1 \\
& IIIB & 1 \\
IIIC & 2 \\
& ER-negative & 1 \\
Grade 3 & 1 \\
& HER2-negative & 1
\end{tabular}

ER: Estrogen receptor; HER2: human epidermal growth factor receptor 2.

control arm or switching to trastuzumab-emtansine (T-DM1) as the experimental arm (6). T-DM1 resulted in improved invasive cancer recurrence and invasive cancer-free survival $(\mathrm{HR}=0.50$, 95\% $\mathrm{CI}=0.39-0.64)$, with an increase in the incidence of thrombocytopenia. These studies demonstrated the utility of changing treatment based on response to NAC. At the same time, the importance of estimating prognosis for individual patients after NAC is being emphasized to guide each patient to the appropriate treatment based on a risk/benefit analysis.

In an effort to evaluate prognosis after NAC, the CSP+EG score, which incorporates the pretreatment clinical stage (cStage) and post-treatment pathological stage (pStage) (CPS score) as well as estrogen receptor (ER) status and tumor nuclear grade (NG), was developed (8). It has been shown that the CPS+EG score better stratifies disease-specific survival (DSS) than does cStage or pStage alone (8). More recently, the Neo-Bioscore, in which HER2 status was incorporated into the CPS+EG score, was shown to stratify DSS even better than the CPS+EG score (Table I) (9).

While more toxic adjuvant treatment has come to be considered for non-pCR cases after the emergence of the trials described above, the utility of the Neo-Bioscore in nonpCR cases or by breast cancer subtype has not been fully evaluated. We therefore explored the utility of the NeoBioscore in guiding post-surgical therapy, particularly in the non-pCR TNBC population.

\section{Patients and Methods}

Eligibility and clinicopathological data collection. A total of 1,096 women diagnosed with primary breast cancer who underwent NAC from October 2005 to April 2017 at the National Cancer Center
Table II. Patient demographics and baseline disease characteristics.

\begin{tabular}{|c|c|c|}
\hline Characteristic & Subgroup & No. of patients (\%) \\
\hline \multirow{6}{*}{$\begin{array}{l}\text { Pretreatment } \\
\text { clinical tumor } \\
\text { stage }\end{array}$} & cT1 & $16(4.9)$ \\
\hline & cT2 & $204(62)$ \\
\hline & cT3 & $54(16)$ \\
\hline & cT4a-c & $50(15)$ \\
\hline & cT4d & $4(1.2)$ \\
\hline & Unknown & $1(0.3)$ \\
\hline \multirow{4}{*}{$\begin{array}{l}\text { Pretreatment } \\
\text { clinical nodal } \\
\text { status }\end{array}$} & $\mathrm{cNO}$ & $102(31)$ \\
\hline & $\mathrm{cN} 1$ & $151(46)$ \\
\hline & $\mathrm{cN} 2$ & $13(4.0)$ \\
\hline & $\mathrm{cN} 3$ & $63(19)$ \\
\hline \multirow{3}{*}{$\begin{array}{l}\text { Tumor nuclear } \\
\text { grade* }\end{array}$} & I & $150(46)$ \\
\hline & II & $90(27)$ \\
\hline & III & $89(27)$ \\
\hline \multirow{2}{*}{$\begin{array}{l}\text { Hormone-receptor } \\
\text { status* }\end{array}$} & Negative & $148(45)$ \\
\hline & Positive & $181(55)$ \\
\hline \multirow[t]{2}{*}{ HER2 status* } & Negative & $237(72)$ \\
\hline & Positive & $92(28)$ \\
\hline \multirow[t]{3}{*}{$\begin{array}{l}\text { Breast cancer } \\
\text { subtype* }\end{array}$} & $\begin{array}{l}\text { Luminal type } \\
\text { (ER+/HER2-) }\end{array}$ & $135(41)$ \\
\hline & HER $2+(E R+$ or ER- $)$ & $92(28)$ \\
\hline & TNBC & $102(31)$ \\
\hline \multirow[t]{2}{*}{ Ki-67* } & $<20 \%$ & $119(36)$ \\
\hline & $\geq 20 \%$ & $210(24)$ \\
\hline \multirow{4}{*}{$\begin{array}{l}\text { Adjuvant } \\
\text { chemotherapy }\end{array}$} & Anthracycline- containing & $283(86)$ \\
\hline & $\begin{array}{l}\text { Non-anthracycline- } \\
\text { containing }\end{array}$ & $46(14)$ \\
\hline & Trastuzumab-containing & $92(28)$ \\
\hline & $\begin{array}{l}\text { Non-trastuzumab- } \\
\text { containing }\end{array}$ & $237(72)$ \\
\hline \multirow{2}{*}{$\begin{array}{l}\text { Type of breast } \\
\text { surgery }\end{array}$} & Lumpectomy & $160(49)$ \\
\hline & Mastectomy & $169(51)$ \\
\hline
\end{tabular}

ER: Estrogen receptor; HER2: human epidermal growth factor receptor 2; TNBC: triple-negative breast cancer. *Core needle biopsy.

Hospital East were listed. Patients were eligible if they were not de novo stage IV cases, and their clinicopathological data including age, cStage, pStage, and NG, as well as ER, HER2, and Ki-67 statuses on diagnostic specimens obtained with core needle biopsies of their primary breast tumors were all available. HER2-positive patients who did not receive trastuzumab along with NAC were excluded. Clinical staging was based on physical examination or ultrasonography of the breast and regional nodal basins at diagnosis, and the latter was prioritized if discrepancy existed. Pathological examination of surgically removed breasts was carried out after sectioning the entire breast into 4-mm-thick slices. Surgically removed axillary lymph nodes were sectioned into 2-mm-thick slices. cStage and pStage were determined according to the eighth Edition of the TNM Classification of Malignant Tumours by the Union for International Cancer Control (10). ER status was classified as positive if $\geq 1 \%$ of tumor cells were ER-positive. HER2 immunohistochemical scoring $(0,1+, 2+$, or $3+)$ was performed according to the manufacturer's recommendations (PATHWAY ${ }^{\circledR}$; Ventana Medical Systems, Tucson, AZ, USA). HER2-positive was defined as a HER2 $3+$ result using PATHWAY ${ }^{\circledR}$ or the presence of HER2 gene amplification using fluorescence in situ hybridization 


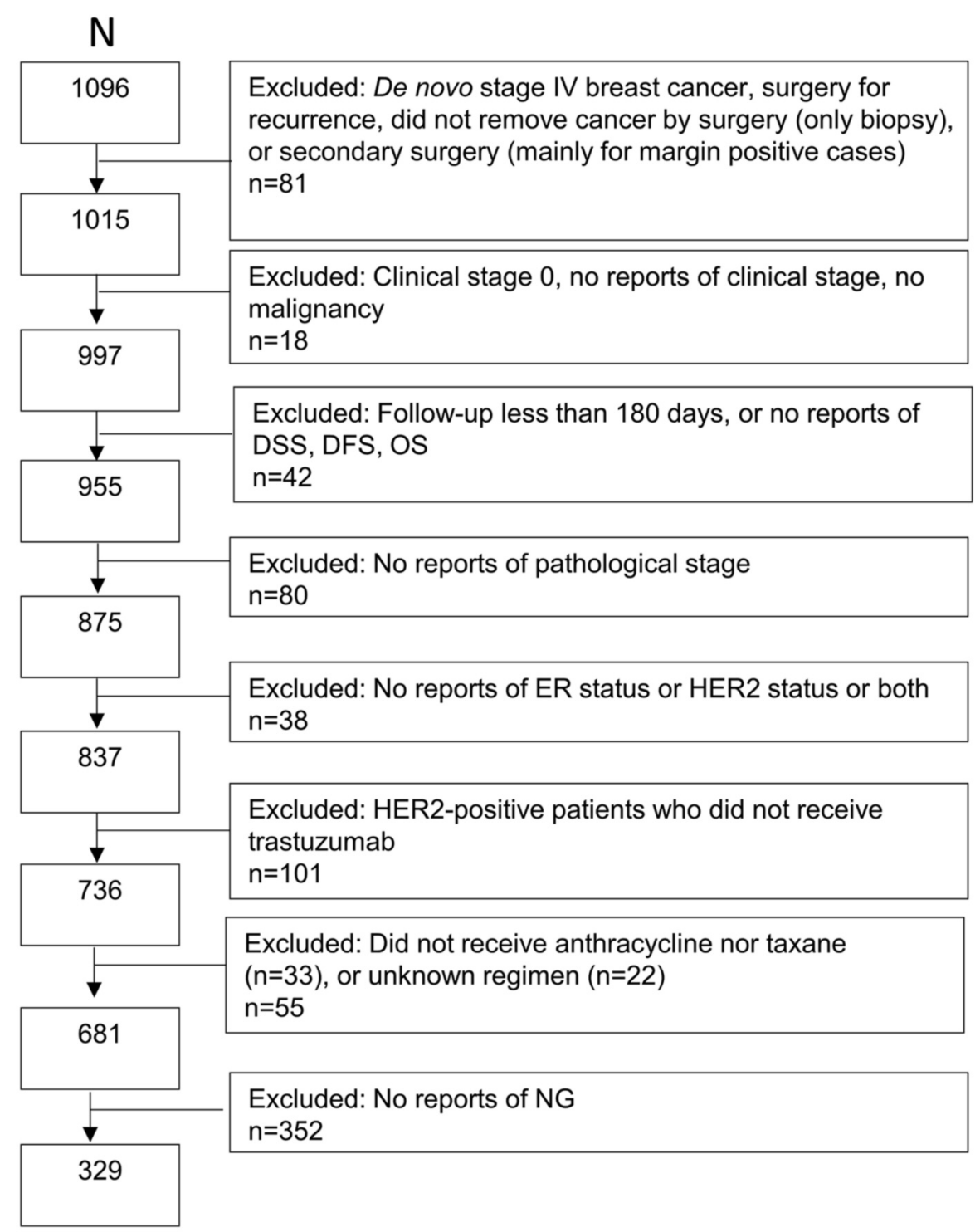

Figure 1. Study CONSORT diagram. DFS: disease-free survival; DSS: disease-specific survival; ER: estrogen receptor; HER2: human epidermal growth factor receptor 2; NG: nuclear grade; OS: overall survival.

assays (HER2 to chromosome 17 centromere ratio $\geq 2$ ), which was typically ordered after obtaining ambiguous HER2 2+ results. NG (score 1-3) was evaluated according to the Elston-Ellis modification of the Scarff-Bloom-Richardson grading system (11). The NeoBioscore was determined for each patient according to a previously published study (9). Point assignments for the Neo-Bioscore staging systems are listed in Table I (9).

After completion of NAC, patients typically underwent local therapy involving either breast-conserving surgery with wholebreast irradiation or mastectomy with or without post-mastectomy radiation therapy, along with axillary evaluation. Patients with hormone receptor-positive tumors routinely received adjuvant endocrine therapy. Patients with HER2-positive disease who received trastuzumab with NAC routinely completed a total of 1 year of trastuzumab therapy.
For DSS and OS analysis, the data-cutoff date was set as March 19, 2019. Date of surgery, presence or absence of local recurrence, distant recurrence, second primary cancer, date of death, date of each event if present, cause of death, the last date of confirming that the patient was alive unless the patient had died, and treatment regimens adopted for NAC were collected from electronic medical records.

Statistical analysis. DSS was defined as the time from the date of surgery to the date of death caused by recurrent breast cancer. OS was defined as the time from the date of surgery to the date of death from any cause. The 5-year DSS and OS rates were estimated using the Kaplan-Meier method (12) for patient subgroups defined using different staging systems, that is, cStage, pStage, and the NeoBioscore. DSS and OS data were analyzed using SPSS 24.0 statistics 
A
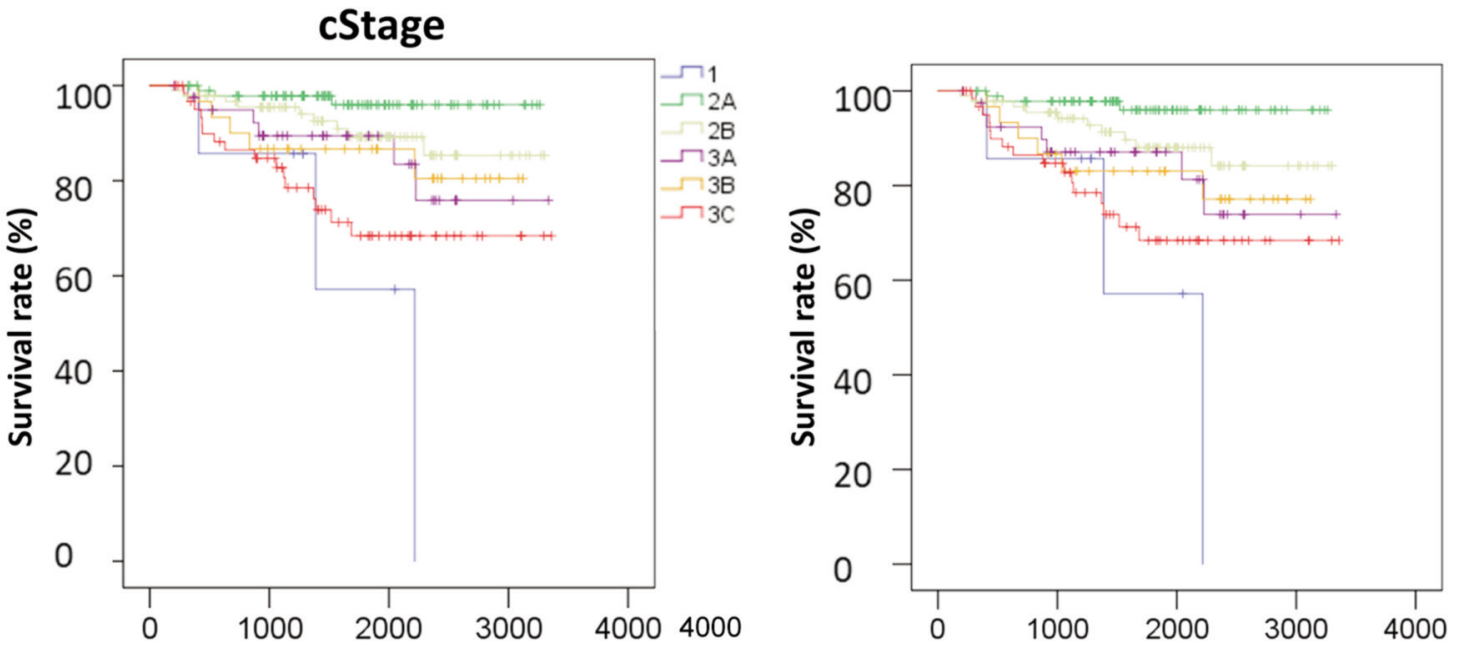

B

pStage
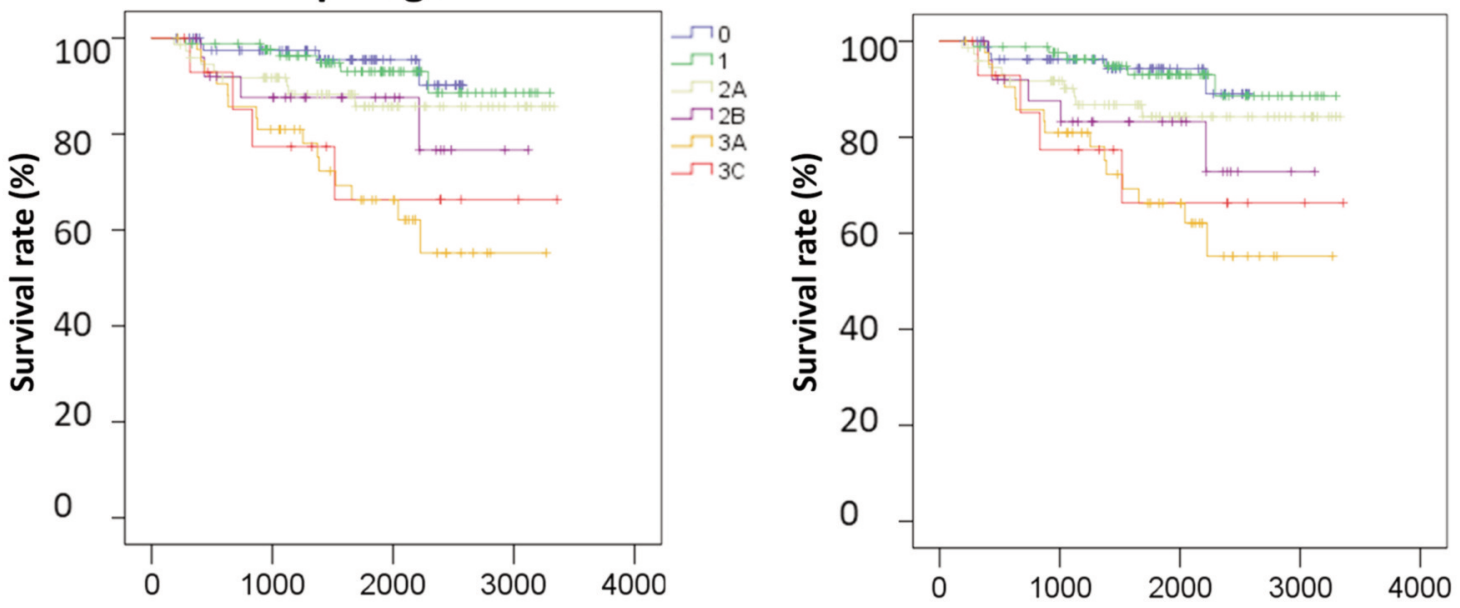

C
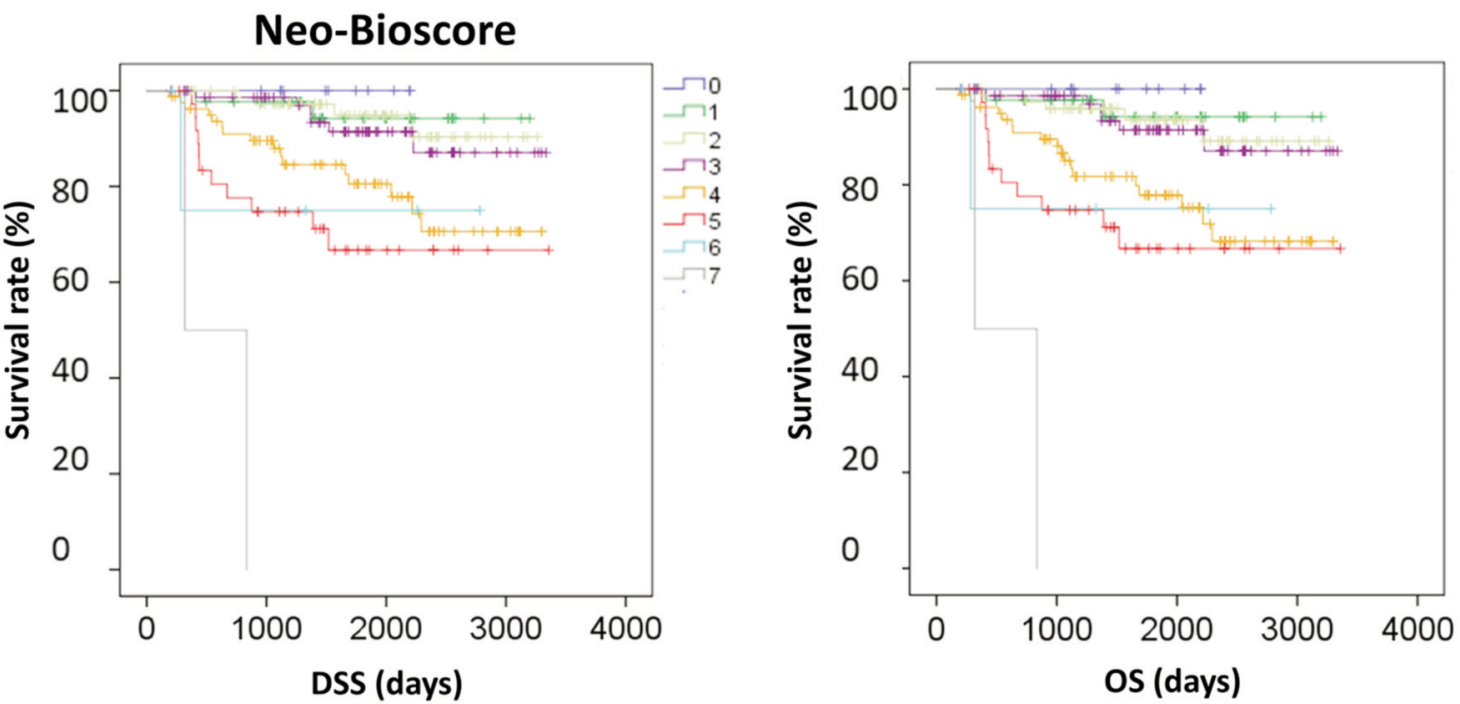

Figure 2. Kaplan-Meier estimates for disease-specific survival (DSS) (left panel) and overall survival (OS) (right panel) for the entire study population based on clinical stage (cStage) (A), pathological stage (pStage) $(B)$, and Neo-Bioscore $(C)$. 
Table III. Five-year disease-specific survival (DSS, \%) outcomes by clinical stage (cStage), pathological stage (pStage), and Neo-Bioscore.

\begin{tabular}{lccccc}
\hline cStage & Median DSS (95\% CI) & pStage & Median DSS (95\% CI) & Neo-Bioscore & Median DSS (95\% CI) \\
\hline 0 & N/A & 0 & $96(90-101)$ & 0 & 100 \\
1 & $57(8-106)$ & 1 & $93(87-99)$ & 2 & $94(86-102)$ \\
$2 \mathrm{~A}$ & $96(91-101)$ & $2 \mathrm{~A}$ & $86(77-95)$ & 3 & $95(89-101)$ \\
2B & $89(82-96)$ & $2 \mathrm{~B}$ & $88(74-101)$ & 4 & $91(84-99)$ \\
3A & $89(80-99)$ & $3 \mathrm{~A}$ & $66(51-81)$ & 6 & $67(71-90)$ \\
$3 \mathrm{~B}$ & $87(75-99)$ & 3B & N/A & 7 & $75(33-117)$ \\
3C & $68(54-81)$ & 3C & $66(38-94)$ & 0 \\
\hline
\end{tabular}

CI: Confidence interval; N/A: not available.

software (IBM, Armonk, NY, USA). The prognostic discriminatory ability of cStage, pStage, and the Neo-Bioscore were evaluated using time-dependent area under the receiver operating characteristic curve (tAUROC), in which a higher integrated tAUROC value indicated better marker performance $(13,14)$. Calculations were performed using SAS 9.4 software (SAS Institute Inc., Cary, NC, USA).

Ethics. A waiver from the need to provide written informed consent for this study was obtained from the Institutional Review Board of the National Cancer Center (2017-431).

\section{Results}

Patient population. From a total of 1,096 listed patients, 721 patients were excluded for reasons shown in the CONSORT diagram (Figure 1). As a result, a total of 329 patients were analyzed. Patient backgrounds are shown in Table II. One hundred and thirty-five (41.0\%), 92 (28.0\%), and 102 $(31.0 \%)$ patients were categorized as having luminal type (ER-positive/HER2-negative), HER2-positive (HER2positive irrespective to ER status), and TNBC, respectively, representing a typical distribution for patients with breast cancer indicated for NAC. Most patients received an anthracycline-containing regimen as their NAC. None of the patients received additional adjuvant chemotherapy.

Prognostic value of the Neo-Bioscore. Prognostic value of cStage, pStage, and the Neo-Bioscore was evaluated. The median follow-up period was 59.2 months. Five-year DSS stratified by cStage, pStage, and the Neo-Bioscore are summarized in Table III. Kaplan-Meier curves for DSS and OS appeared to be better separated using the Neo-Bioscore than using cStage or pStage (Figure 2). The integrated tAUROC of the Neo-Bioscore for DSS and OS was higher than that for cStage and pStage (integrated tAUROC for DSS; cStage vs. pStage vs. Neo-Bioscore, 0.6441 vs. 0.6751 vs. 0.7515, respectively) (integrated tAUROC for OS; cStage vs. pStage vs. Neo-Bioscore, 0.6448 vs. 0.6573 vs. 0.7375 , respectively) (Figure 3). This indicates the superior discriminatory ability of the Neo-Bioscore to cStage or pStage for predicting prognosis after NAC.
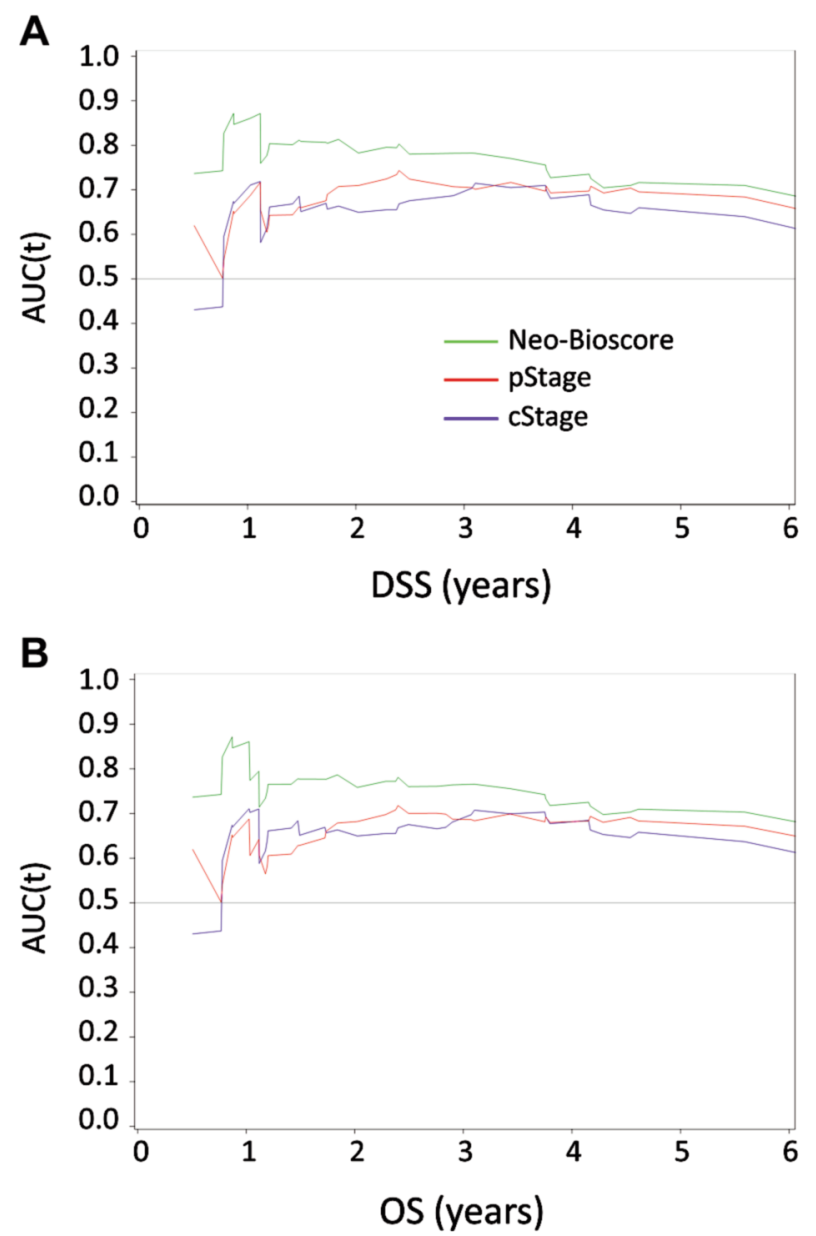

Figure 3. Time-dependent receiver operating characteristic (ROC) curves for clinical stage (cStage), pathological stage (pStage), and NeoBioscore in predicting disease-specific survival (DSS) (A) and overall survival $(O S)(B)$. AUC $(t)$ : Time-dependent area under the ROC curve.

Prognostic value of the Neo-Bioscore in TNBC. We next evaluated the prognostic value of the Neo-Bioscore separately in TNBC because stratifying post-NAC prognosis is particularly important for this subtype, as highlighted in the 

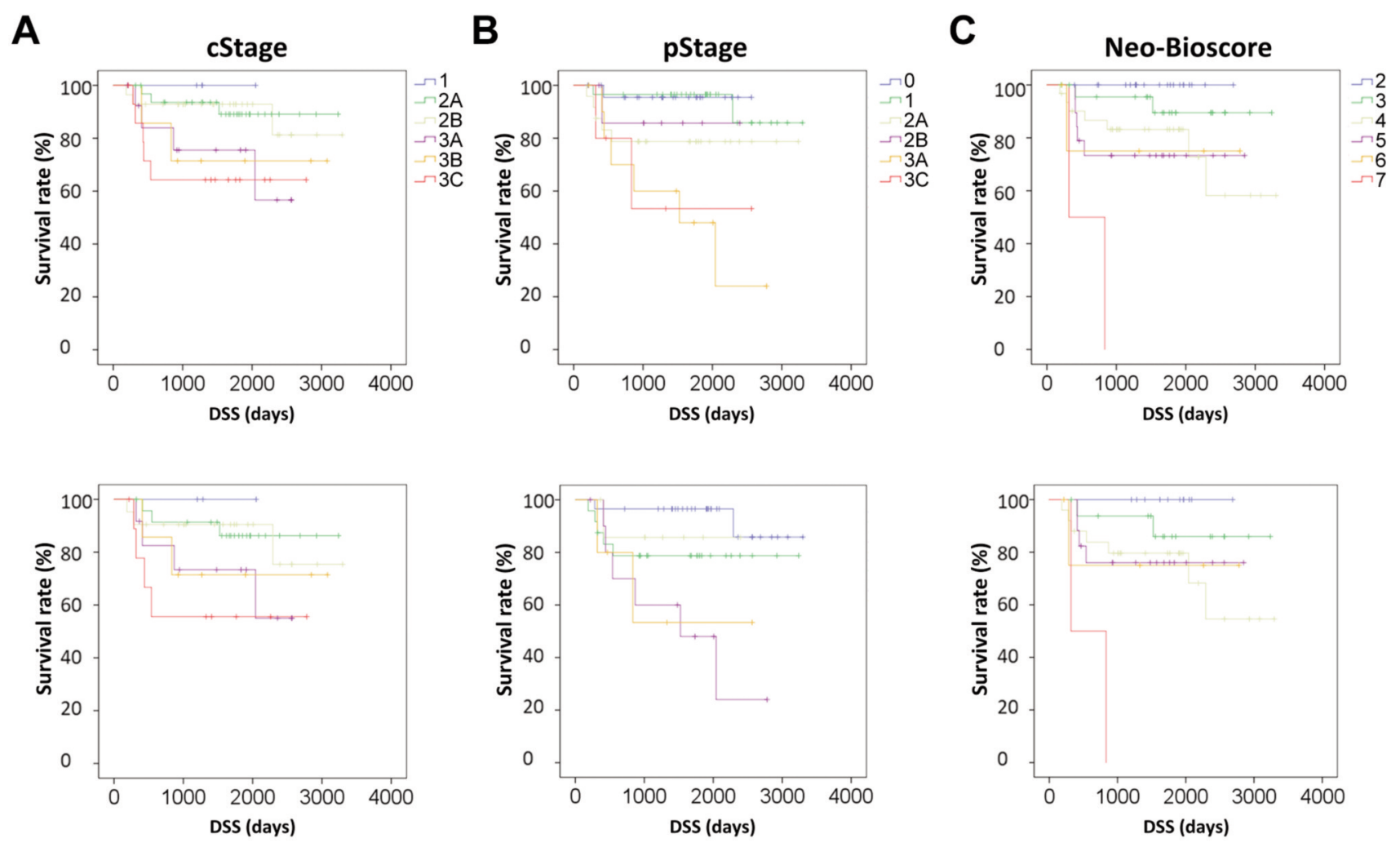

Figure 4. Kaplan-Meier estimates for disease-specific survival (DSS) in the triple-negative breast cancer study population, including those with pathological complete response ( $p C R)$ (upper panel) and those with non-pCR (lower panel), based on clinical stage (cStage) (A), pathological stage (pStage) (B), and Neo-Bioscore $(C)$.

CREATE X trial (see above). Even though superiority of the Neo-Bioscore to cStage or pStage in stratifying DSS was not clearly demonstrated (integrated tAUROC for DSS; cStage $v s$. pStage vs. Neo-Bioscore, 0.6756 vs. 0.7116 vs. 0.7296 , respectively), the Neo-Bioscore appeared to perform well in selecting patients with an excellent prognosis after NAC (Figures 4 and 5, upper panels). Of 25 patients with TNBC who achieved pCR, only one died with recurrent breast cancer. Even when excluding these pCR cases, that is, for the 'CREATE X population, 11 patients with a Neo-Bioscore of 2, the lowest score that can be determined in TNBC, had no DSS or OS events (Figures 4 and 5, lower panels). For the NeoBioscore to be 2, as in non-pCR TNBC cases, cStage and pStage had to be I or IIA and I, respectively. Three out of 27 (11.1\%) patients with cStage I or IIA and two out of $29(6.9 \%)$ patients with pStage I died with recurrent breast cancer, indicating an advantage of the Neo-Bioscore in selecting patients with an excellent prognosis despite having a non-pCR.

\section{Discussion}

In this study, using a data set of Japanese patients, we demonstrated that the Neo-Bioscore staging can provide better prognostic information about DSS and OS than clinical or pathological staging. Additionally, we found that the ability of the Neo-Bioscore in stratifying prognosis was maintained even when excluding pCR cases in the TNBC subtype. Finally, we found that for highly selected patients with TNBC with a NeoBioscore of 2, omitting capecitabine may be considered despite the presence of residual disease after NAC.

After publication of the original article on the NeoBioscore (9), several studies validated its usefulness in different cohorts. One large cohort study $(n=12,002)$ utilizing the National Cancer Database showed that the Neo-Bioscore was better in discriminating OS over CPS+EG, clinical staging, and pathological staging (15). A single-institutional study from Peking University, China, concluded that the CPS+EG score and the Neo-Bioscore were both better than clinical staging in discriminating DFS, DSS, and OS, although they failed to show an advantage of the NeoBioscore over pathological staging or the CPS+EG score, possibly because of the small sample size $(n=403)(16)$. Our current results are consistent with these studies based on real-world data, although we did not focus on comparison of the Neo-Bioscore with the CPS+EG score because of the small sample size. 

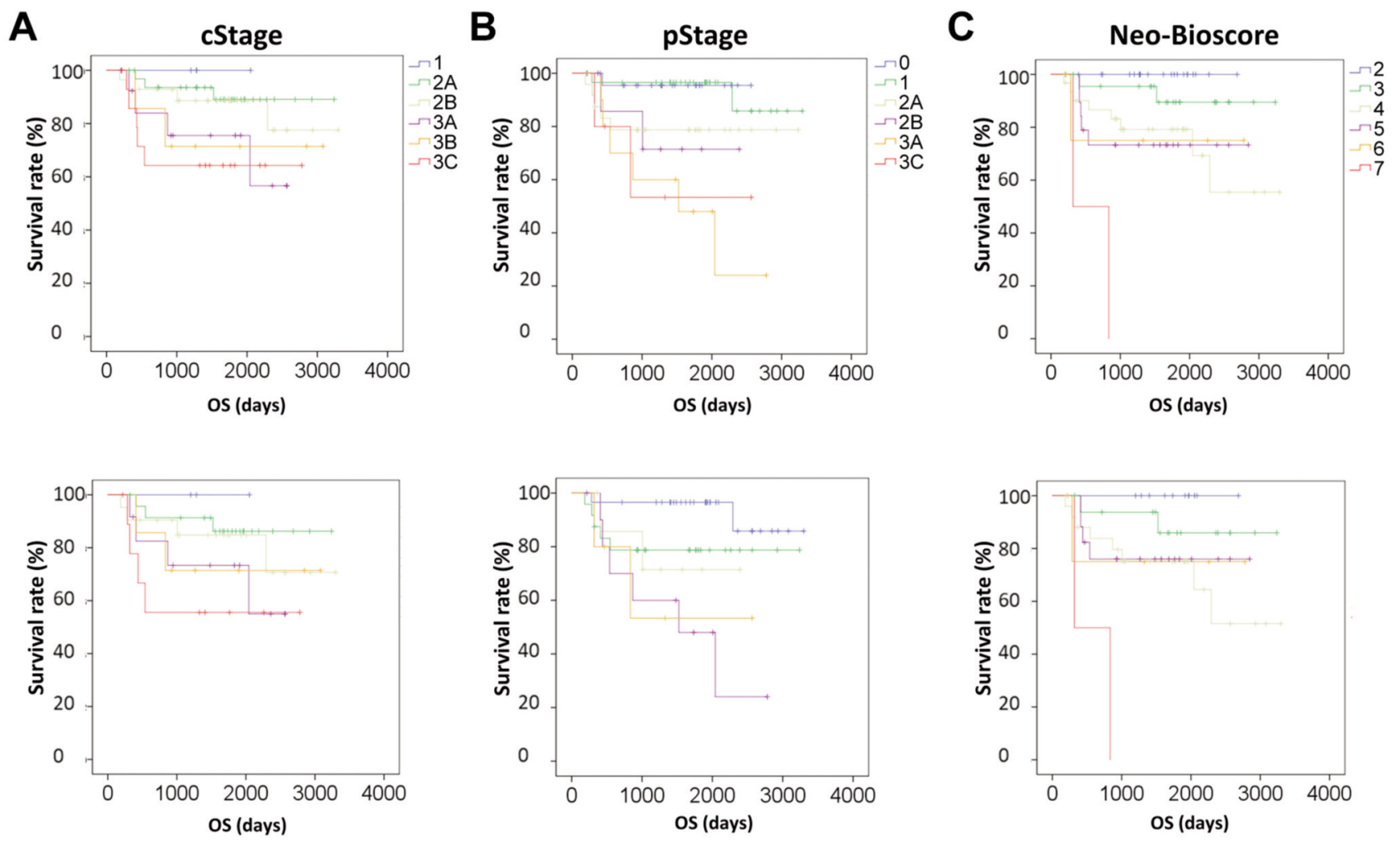

Figure 5. Kaplan-Meier estimates for overall survival (OS) in the triple-negative breast cancer study population, including those with pathological complete response ( $p C R)$ (upper panel) and those with non-pCR (lower panel), based on clinical stage (cStage) (A), pathological stage (pStage) $(B)$, and Neo-Bioscore $(C)$.

The prognostic value of the CPS+EG score, but not of NeoBioscore staging, has been evaluated according to breast cancer subtype. A study involving 6,637 unselected patients and 2,454 patients with hormone receptor-positive/HER2-negative (luminal type) tumors who received anthracycline/taxane-based NAC within eight prospective randomized neoadjuvant trials conducted by the German Breast Group showed that while more patients with luminal-type tumors had CPS+EG 0-1 scores $(44 \%)$ than the subtype-unselected entire population (33\%), the 5-year DFS and OS for each individual score was identical for these populations (17). The CPS+EG score was also shown to discriminate prognosis similarly in both the TNBC and the entire HER2-negative population in a study involving 4,812 patients who were given NAC within nine prospective randomized neoadjuvant trials conducted by the German Breast Group (18). Consistent with these studies, the discriminatory capacity of the Neo-Bioscore appeared to be sustained when limiting it to TNBC in our current study.

While pCR is an accepted indicator of a good prognosis, the prognosis of patients not achieving a pCR is less defined but more clinically relevant after the emergence of results of the CREATE X and KATHERINE trials. In particular, it is of great importance to identify patient groups with excellent prognosis, despite non-pCR, in order to avoid more toxic and more costly post-surgical treatment. Even though the NeoBioscore was developed including patients who achieved pCR, we showed that the Neo-Bioscore stratified patient prognosis even after excluding pCR cases in the TNBC population (Figure 4 lower panel). Additionally, we suggest that patients with TNBC with a Neo-Bioscore of 2 may avoid adjuvant capecitabine because we observed no DSS or OS events for this particular cohort (Figures 4 and 5). A similar attempt at identifying patients with a good prognosis despite having a non-pCR was made in a recently published study, in which it was found that patients with non-pCR had a 5-year recurrence-free survival comparable to patients with $\mathrm{pCR}$ using the combination of the Neo-Bioscore, residual cancer burden, and Neoadjuvant Response Index (19). Conversely, the above-mentioned study from the German Breast Group showed that even non-pCR with a CPS+EG score of 1, the lowest for TNBC and equivalent to a Neo-Bioscore of 2, cStage I-IIA, and pStage I, had unsatisfactory 5-year-DFS of $77.5 \%$ (18). The cause of these discrepant results is not clear but may be related to the differential biology of TNBC between different ethnicities or a difference in the stringency of the pathological examination between facilities. 
Our study had some limitations. Firstly, this was a retrospective analysis with a relatively small sample size from a single institute, which limits its statistical power. Secondly, in our study, we were unable to apply the NeoBioscore to patients with non-pCR HER2-positive disease, who are recommended T-DM1, because there were very few DSS events (five DSS events in 53 non-pCR HER2positive cases). Using a database that includes more patients may overcome these limitations, and we are attempting to validate our current findings in a nationwide study in Japan.

In conclusion, this study showed that the Neo-Bioscore staging system can improve the prognostic stratification of patients with patients after NAC for breast cancer over cStage and pStage. Moreover, the Neo-Bioscore may enable the identification of patients with non-pCR TNBC who can avoid additional adjuvant chemotherapy, although large-scale validation studies are necessary.

\section{Conflicts of Interest}

The Authors declare that there are no conflicts of interest regarding this study.

\section{Authors' Contributions}

All Authors contributed to the study conception and design. Data collection and analysis were performed by Yoriko Hasegawga, Toru Mukohara and Mototsugu Shimokawa. The first draft of the article was written by Yoriko Hasegawa and Toru Mukohara, and all Authors commented on previous versions of the article, and read and approved the final article.

\section{Acknowledgements}

The Authors thank Ms. Ryoko Wanikawa for her excellent work in data collection. They also thank Mark Abramovitz, Ph D, from Edanz Group (https://en-author-services.edanzgroup.com/) for editing a draft of this article.

\section{References}

1 Mauri D, Pavlidis N and Ioannidis JP: Neoadjuvant versus adjuvant systemic treatment in breast cancer: A meta-analysis. J Natl Cancer Inst 97(3): 188-194, 2005. PMID: 15687361. DOI: 10.1093/jnci/dji021

2 Scholl SM, Fourquet A, Asselain B, Pierga JY, Vilcoq JR, Durand JC, Dorval T, Palangie T, Jouve M, Beuzeboc P, GarcioGiralt E, Salmon RJ, de la Rochefordière A, Campana F and Pouillart P: Neoadjuvant versus adjuvant chemotherapy in premenopausal patients with tumours considered too large for breast conserving surgery: Preliminary results of a randomised trial: S6. Eur J Cancer 30A(5): 645-652, 1994. PMID: 8080680. DOI: $10.1016 / 0959-8049(94) 90537-1$

3 van der Hage JA, van de Velde CJ, Julien JP, Tubiana-Hulin M, Vandervelden C and Duchateau L: Preoperative chemotherapy in primary operable breast cancer: Results from the European Organization for Research and Treatment of Cancer trial 10902. J Clin Oncol 19(22): 4224-4237, 2001. PMID: 11709566. DOI: 10.1200/JCO.2001.19.22.4224

4 Cortazar P, Zhang L, Untch M, Mehta K, Costantino JP, Wolmark N, Bonnefoi H, Cameron D, Gianni L, Valagussa P, Swain SM, Prowell T, Loibl S, Wickerham DL, Bogaerts J, Baselga J, Perou C, Blumenthal G, Blohmer J, Mamounas EP, Bergh J, Semiglazov V, Justice R, Eidtmann H, Paik S, Piccart M, Sridhara R, Fasching PA, Slaets L, Tang S, Gerber B, Geyer CE, Jr., Pazdur R, Ditsch N, Rastogi P, Eiermann W and von Minckwitz G: Pathological complete response and long-term clinical benefit in breast cancer: The CTNEOBC pooled analysis. Lancet 384(9938): 164-172, 2014. PMID: 24529560. DOI: $10.1016 / \mathrm{S} 0140-6736(13) 62422-8$

5 Masuda N, Lee SJ, Ohtani S, Im YH, Lee ES, Yokota I, Kuroi K, Im SA, Park BW, Kim SB, Yanagita Y, Ohno S, Takao S, Aogi K, Iwata H, Jeong J, Kim A, Park KH, Sasano H, Ohashi $\mathrm{Y}$ and Toi M: Adjuvant capecitabine for breast cancer after preoperative chemotherapy. N Engl J Med 376(22): 2147-2159, 2017. PMID: 28564564. DOI: 10.1056/NEJMoa1612645

6 von Minckwitz G, Huang CS, Mano MS, Loibl S, Mamounas EP, Untch M, Wolmark N, Rastogi P, Schneeweiss A, Redondo A, Fischer HH, Jacot W, Conlin AK, Arce-Salinas C, Wapnir IL, Jackisch C, DiGiovanna MP, Fasching PA, Crown JP, Wulfing P, Shao Z, Rota Caremoli E, Wu H, Lam LH, Tesarowski D, Smitt M, Douthwaite H, Singel SM, Geyer CE, Jr. and Investigators $\mathrm{K}$ : Trastuzumab emtansine for residual invasive HER2-positive breast cancer. N Engl J Med 380(7): 617-628, 2019. PMID: 30516102. DOI: 10.1056/NEJMoa1814017

7 National Comprehensive Cancer Network: NCCN Clinical Practice Guidelines in Oncology (NCCN guidelines $\left.{ }^{\circledR}\right)$, Breast Cancer, Version 4. 2020.

8 Jeruss JS, Mittendorf EA, Tucker SL, Gonzalez-Angulo AM, Buchholz TA, Sahin AA, Cormier JN, Buzdar AU, Hortobagyi GN and Hunt KK: Combined use of clinical and pathologic staging variables to define outcomes for breast cancer patients treated with neoadjuvant therapy. J Clin Oncol 26(2): 246-252, 2008. PMID: 18056680. DOI: 10.1200/JCO.2007.11.5352

9 Mittendorf EA, Vila J, Tucker SL, Chavez-MacGregor M, Smith BD, Symmans WF, Sahin AA, Hortobagyi GN and Hunt KK: The neo-bioscore update for staging breast cancer treated with neoadjuvant chemotherapy: Incorporation of prognostic biologic factors into staging after treatment. JAMA Oncol 2(7): 929-936, 2016. PMID: 26986538. DOI: 10.1001/jamaoncol.2015.6478

10 TNM Classification of Malignant Tumours, Eighth Edition. Brierley JD, Gospodarowicz MK and Wittekind C (eds.). WileyBlackwell, 2017.

11 Elston CW and Ellis IO: Pathological prognostic factors in breast cancer. I. The value of histological grade in breast cancer: Experience from a large study with long-term follow-up. Histopathology 19(5): 403-410, 1991. PMID: 1757079. DOI: 10.1111/j.1365-2559.1991.tb00229.x

12 Kaplan EL and Meier P: Nonparametric estimation from incomplete observations. J Amer Statist 53: 457-481, 1958. DOI: $10.2307 / 2281868$

13 Chambless LE and Diao G: Estimation of time-dependent area under the ROC curve for long-term risk prediction. Stat Med 25(20): 3474-3486, 2006. PMID: 16220486. DOI: 10.1002/ sim.2299 
14 Heagerty PJ, Lumley T and Pepe MS: Time-dependent ROC curves for censored survival data and a diagnostic marker. Biometrics 56(2): 337-344, 2000. PMID: 10877287. DOI: 10.1111/j.0006-341x.2000.00337.x

15 Bergquist JR, Murphy BL, Storlie CB, Habermann EB and Boughey JC: Incorporation of treatment response, tumor grade and receptor status improves staging quality in breast cancer patients treated with neoadjuvant chemotherapy. Ann Surg Oncol 24(12): 3510-3517, 2017. PMID: 28828583. DOI: 10.1245/ s10434-017-6010-4

16 Xu L, Duan X, Zhou B, Liu Y, Ye J, Liu Z, Ma C, Zhang H, Zhang S, Zhang L, Zhao J and Cheng Y: Validation of the $\mathrm{CPS}+\mathrm{EG}$ and Neo-Bioscore staging systems after preoperative systemic therapy for breast cancer in a single center in China. Breast 40: 29-37, 2018. PMID: 29677568. DOI: 10.1016/ j.breast.2018.03.010

17 Marme F, Lederer B, Blohmer JU, Costa SD, Denkert C, Eidtmann H, Gerber B, Hanusch C, Hilfrich J, Huober J, Jackisch C, Kummel S, Loibl S, Paepke S, Untch M, von Minckwitz G and Schneeweiss A: Utility of the CPS+EG staging system in hormone receptor-positive, human epidermal growth factor receptor 2-negative breast cancer treated with neoadjuvant chemotherapy. Eur J Cancer 53: 65-74, 2016. PMID: 26693900. DOI: $10.1016 /$ j.ejca.2015.09.022
18 Marme F, Solbach C, Michel L, Fasching PA, Schneeweiss A, Blohmer JU, Rezai M, Huober J, Jackisch C, Nekljudova V, Link T, Rhiem K, Denkert C, Hanusch C, Tesch H, Lederer B, Loibl S and Untch M: Utility of the CPS plus EG scoring system in triple-negative breast cancer treated with neoadjuvant chemotherapy. Ann Oncol 30(S5): 58, 2019.

19 Steenbruggen TG, van Seijen M, Janssen LM, van Ramshorst MS, van Werkhoven E, Vrancken Peeters M, Wesseling J, Lips EH and Sonke GS: Prognostic value of residual disease after neoadjuvant therapy in HER2-positive breast cancer evaluated by residual cancer burden, neoadjuvant response index, and neobioscore. Clin Cancer Res 25(16): 4985-4992, 2019. PMID: 31076546. DOI: 10.1158/1078-0432.CCR-19-0560

Received November 10, 2020

Revised December 14, 2020

Accepted December 15, 2020 